\title{
SCREENING OF BASIDIOMYCETES FOR THE PRODUCTION OF EXOPOLYSACCHARIDE AND BIOMASS IN SUBMERGED CULTURE
}

\author{
Rosana Maziero $^{1^{*},}$ Valeria Cavazzoni $^{2}$, Vera Lúcia Ramos Bononi ${ }^{1}$ \\ ${ }^{1}$ Instituto de Botânica, São Paulo, SP, Brasil. ${ }^{2}$ Dipartimento di Scienze e Tecnologie Alimentari e \\ Microbiologiche, Sez. Microbiologia Industriale, Milano, Italy
}

Submitted: September 23, 1997; Returned to authors for corrections: February 06, 1998; Approved: November 12, 1998.

\begin{abstract}
Fifty-six strains of Basidiomycetes, including native Brazilian fungi isolated from different ecosystems and edible mushrooms, were screened for production of exopolysaccharides and biomass in submerged culture. Agaricus sp. (CCB 280) and Oudemansiella canarii (Jungh.) Hohn (CCB 179) were the highest exopolysaccharide producers (6.01 and 3.54 g dry w./1 respectively) after 7 days of incubation. The best producer of biomass was Schizophyllum commune Fr.:Fr. (CCB 473) with $16.68 \mathrm{~g}$ dry w./l in 14 days of incubation. When the culture filtrate was submitted to freezing prior to polysaccharide precipitation, a gelatinous fraction was formed.
\end{abstract}

Key words: Basidiomycetes, exopolysaccharide, biomass, submerged culture

\section{INTRODUCTION}

Following previous work regarding collection, identification and isolation of native Brazilian Basidiomycetes in pure culture, investigations were carried out concerning their utilization in biotechnological processes such as lignin and recalcitrant substances degradation, soil bioremediation, edible fungal biomass and metabolites production.

Basidiomycetes have been studied extensively for their capacity of degradation. The so-called white rot fungi, which degrade lignin, have this peculiar capacity that leads to research on degradation of xenobiotics. In addition to enzymes, there is evidence that the extracellular polysaccharides produced by these lignocellulolitic fungi play an important role in the process $(6,16)$. These exopolysaccharides can immobilize the exocellular enzymes. According to Catley (3), the gel formed by these biopolymers prevents the hyphal dehydration, permits cell adherence to other cells or to surfaces and could possibly select molecules from the environment.

A practical aspect of the study and characterization of fungal exopolysaccharide is the availability of data for the investigation of its physiological and ecological importance. In addition, this biopolymer may have potential industrial applications. An example is the exopolysaccharide known as schizophyllan that is produced by the Basidiomycete Schizophyllum commune. This polymer is a $\beta-(1 \rightarrow 3),(1 \rightarrow 6)$-glucan, soluble in water, that forms a viscous solution with high thermal stability. It is already used in commercial areas.

Another possible application of these biopolymers is in human health. There is intensive research on fungal polysaccharides as antitumor agents $(8,9)$.

\footnotetext{
* Corresponding author. Mailing address: Instituto de Botânica, Caixa Postal 4005, CEP 01061-970, São Paulo, SP, Brasil. Fax: (+5511) $577-$ 3678.E-mail: rosana@tin.it
} 
The fungal biomass can have various uses, which is an advantage as far as the fermentation is concerned because the process residue is reduced (14). Possible uses for this biomass are food or feed in the form of protein supplement or source of lipids. It can also be used for the extraction of flavours (10) and other metabolites, such as enzymes and polysaccharides. The most recent utilization of fungal biomass is for wound healing. According to Hamlyn and Schmidt (7), chitin, that has a healing capacity, is already in the fibrous form when extracted from the fungal cell wall. This might facilitate its manipulation.

The aim of this work was to screen 56 strains of Basidiomycetes for exopolysaccharide and biomass production in submerged culture contributing to the study of the potentiality of the Brazilian mycobiota.

\section{MATERIALS AND METHODS}

Microorganisms. 48 strains of native Brazilian Basidiomycetes and 8 strains of commercial edible mushrooms, corresponding to 51 different species belonging to 42 genera, were screened. The pure cultures came from the Culture Collection of Basidiomycetes (CCB) of the Instituto de Botânica São Paulo - Brazil, and are shown in Table 1.
Liquid culture medium (g/l): Peptone 1.0; yeast extract 2.0; $\mathrm{K}_{2} \mathrm{HPO}_{4} 1.0 ; \mathrm{MgSO}_{4} \cdot 7 \mathrm{H}_{2} \mathrm{O} 0.2 ;\left(\mathrm{NH}_{4}\right)_{2} \mathrm{SO}_{4}$ 5.0; glucose 39.0; $\mathrm{pH}$ 6.0. This medium was selected in preliminary studies as adequate for exopolysaccharide production by Basidiomycetes (4).

Erlenmeyer flasks containing $100 \mathrm{ml}$ of sterilized culture medium were inoculated with the suspension in sterile water of fungal mycelium grown on two potato dextrose agar slants. Incubation was done at $25^{\circ} \mathrm{C}$ on shaker at $150 \mathrm{rpm}$.

Screening. For the screening, the incubation times were 7 and 14 days. The culture was filtered to separate fungal biomass, which was washed twice with distilled water and quantified as dry weight $\left(105^{\circ} \mathrm{C}\right.$ to constant weight). Isopropanol was added to the culture filtrate $(1: 1 \mathrm{v} / \mathrm{v})$ and after $24 \mathrm{~h}$ at $4^{\circ} \mathrm{C}$ the precipitated biopolymer was separated by centrifugation $(8,000$ rpm for 10 minutes) and also quantified as dry weight.

Glucose assay. The residual glucose content of the culture filtrate was determined with a colorimetric method (17).

Chemicals used were produced by: E. Merck GmbH, Darmstadt, Germany; BDH Chemicals Ltd, Poole, England; Boehringer Manheim GmbH; Fluka Chemie AG., Buchs, Switzerland; DIFCO Laboratories, Detroit, U.S.A. and A. Constantino \& C. s.p.a., Favria, Italy.

Table 1 - Results of the screening for the production of exopolysaccharide $\left(\mathrm{P}_{\mathrm{p}}\right)$ and biomass $\left(\mathrm{P}_{\mathrm{x}}\right)$, with the conversion yield of glucose in polymer $\left(\mathrm{Y}_{\mathrm{p} / \mathrm{s}}\right)$, in biomass $\left(\mathrm{Y}_{\mathrm{x} / \mathrm{s}}\right)$ and the specific yield $\left(\mathrm{Y}_{\mathrm{e}}\right)$.

\begin{tabular}{llcccccc}
\hline CCB & STRAIN & DAY & $\begin{array}{c}\mathrm{P}_{\mathrm{x}} \\
\mathrm{g} \mathrm{dry} \mathrm{w./1}\end{array}$ & $\mathrm{Y}_{\mathrm{x} / \mathrm{s}}$ & $\begin{array}{c}\mathrm{P}_{\mathrm{p}} \\
\mathrm{g} \mathrm{dry} \mathrm{w./1}\end{array}$ & $\mathrm{Y}_{\mathrm{p} / \mathrm{s}}$ & $\mathrm{Y}_{\mathrm{e}}$ \\
\hline 041 & Agaricus xanthodermus & 7 & 0.88 & 0.098 & 1.61 & .0179 & 1.830 \\
& $*$ & 14 & 0.92 & 0.137 & 1.39 & 0.207 & 1.511 \\
280 & Agaricus sp. & 7 & 1.64 & 0.208 & 6.01 & 0.761 & 3.665 \\
& & 14 & 3.09 & 0.322 & 1.36 & 0.142 & 0.440 \\
211 & Agrocybe platensis & 7 & 8.37 & 0.406 & 1.00 & 0.048 & 0.120 \\
& & 14 & 10.18 & 0.318 & 1.33 & 0.042 & 0.131 \\
392 & Antrodiella ginestae & 7 & 6.28 & 0.262 & 0.49 & 0.020 & 0.078 \\
& & 14 & 7.15 & 0.248 & 0.64 & 0.022 & 0.090 \\
045 & Auricularia fuscosuccinea & 7 & 1.20 & 0.200 & 0.54 & 0.090 & 0.450 \\
& $*$ & 14 & 3.62 & 0.266 & 1.10 & 0.081 & 0.304 \\
173 & Calvatia cyathiformis & 7 & 1.10 & 0.157 & 2.05 & 0.293 & 1.864 \\
& $*$ & 14 & 0.70 & 0.082 & 0.72 & 0.085 & 1.029 \\
191 & Climacodon pulcherrimus & 7 & 0.34 & 0.046 & 0.75 & 0.101 & 2.206 \\
& & 14 & 0.63 & 0.066 & 1.03 & 0.107 & 1.635 \\
\hline
\end{tabular}


(continuação...)

\begin{tabular}{|c|c|c|c|c|c|c|c|}
\hline $\mathrm{CCB}$ & STRAIN & DAY & $\begin{array}{c}\mathrm{P}_{\mathrm{x}} \\
\text { g dry w./1 } \\
\end{array}$ & $\mathrm{Y}_{\mathrm{x} / \mathrm{s}}$ & $\begin{array}{c}\mathrm{P}_{\mathrm{p}} \\
\text { g dry w./1 } \\
\end{array}$ & $\mathrm{Y}_{\mathrm{p} / \mathrm{s}}$ & $\mathrm{Y}_{\mathrm{e}}$ \\
\hline 111 & Coprinus comatus & $\begin{array}{c}7 \\
14\end{array}$ & $\begin{array}{l}5.18 \\
7.56\end{array}$ & $\begin{array}{l}0.395 \\
0.450\end{array}$ & $\begin{array}{l}1.11 \\
1.53\end{array}$ & $\begin{array}{l}0.085 \\
0.091\end{array}$ & $\begin{array}{l}0.214 \\
0.202\end{array}$ \\
\hline 513 & Flammulina velutipes & $\begin{array}{c}7 \\
14\end{array}$ & $\begin{array}{l}4.86 \\
8.28\end{array}$ & $\begin{array}{l}0.273 \\
0.213\end{array}$ & $\begin{array}{l}1.05 \\
1.74\end{array}$ & $\begin{array}{l}0.059 \\
0.045\end{array}$ & $\begin{array}{l}0.216 \\
0.210\end{array}$ \\
\hline 214 & Fomitopsis spraguei & $\begin{array}{c}7 \\
14\end{array}$ & $\begin{array}{l}2.91 \\
2.38\end{array}$ & $\begin{array}{l}0.239 \\
0.165\end{array}$ & $\begin{array}{l}0.21 \\
0.28\end{array}$ & $\begin{array}{l}0.017 \\
0.019\end{array}$ & $\begin{array}{l}0.072 \\
0.118\end{array}$ \\
\hline 168 & Ganoderma australe & $\begin{array}{c}7 \\
14\end{array}$ & $\begin{array}{l}14.75 \\
15.02\end{array}$ & $\begin{array}{l}0.382 \\
0.387\end{array}$ & $\begin{array}{l}2.64 \\
2.04\end{array}$ & $\begin{array}{l}0.068 \\
0.053\end{array}$ & $\begin{array}{l}0.179 \\
0.136\end{array}$ \\
\hline 323 & $\begin{array}{l}\text { Ganoderma lipsiensis } \\
*\end{array}$ & $\begin{array}{c}7 \\
14\end{array}$ & $\begin{array}{r}7.29 \\
13.24\end{array}$ & $\begin{array}{l}0.361 \\
0.404\end{array}$ & $\begin{array}{l}0.98 \\
2.75\end{array}$ & $\begin{array}{l}0.049 \\
0.084\end{array}$ & $\begin{array}{l}0.134 \\
0.208\end{array}$ \\
\hline 177 & Gloeophyllum striatum & $\begin{array}{c}7 \\
14\end{array}$ & $\begin{array}{l}5.70 \\
8.60\end{array}$ & $\begin{array}{l}0.533 \\
0.422\end{array}$ & $\begin{array}{l}0.20 \\
0.53\end{array}$ & $\begin{array}{l}0.019 \\
0.026\end{array}$ & $\begin{array}{l}0.035 \\
0.062\end{array}$ \\
\hline 188 & Gloeophyllum striatum & $\begin{array}{c}7 \\
14\end{array}$ & $\begin{array}{l}4.56 \\
8.06\end{array}$ & $\begin{array}{l}0.356 \\
0.593\end{array}$ & $\begin{array}{l}0.19 \\
0.15\end{array}$ & $\begin{array}{l}0.015 \\
0.011\end{array}$ & $\begin{array}{l}0.042 \\
0.019\end{array}$ \\
\hline 249 & Gymnopilus sp. & $\begin{array}{c}7 \\
14\end{array}$ & $\begin{array}{l}2.98 \\
7.00\end{array}$ & $\begin{array}{l}0.339 \\
0.432\end{array}$ & $\begin{array}{l}0.41 \\
1.08\end{array}$ & $\begin{array}{l}0.047 \\
0.067\end{array}$ & $\begin{array}{l}0.138 \\
0.154\end{array}$ \\
\hline 289 & Hydnopolyporus fimbriatus & $\begin{array}{c}7 \\
14\end{array}$ & $\begin{array}{l}2.40 \\
3.71\end{array}$ & $\begin{array}{l}0.189 \\
0.277\end{array}$ & $\begin{array}{l}0.26 \\
0.51\end{array}$ & $\begin{array}{l}0.020 \\
0.038\end{array}$ & $\begin{array}{l}0.108 \\
0.137\end{array}$ \\
\hline 160 & Hypochnicium sp. & $\begin{array}{c}7 \\
14\end{array}$ & $\begin{array}{l}11.24 \\
10.30\end{array}$ & $\begin{array}{l}0.420 \\
0.380\end{array}$ & $\begin{array}{l}1.50 \\
1.20\end{array}$ & $\begin{array}{l}0.056 \\
0.044\end{array}$ & $\begin{array}{l}0.133 \\
0.116\end{array}$ \\
\hline 207 & Inonotus ludovicianus & $\begin{array}{c}7 \\
14\end{array}$ & $\begin{array}{l}0.99 \\
2.56\end{array}$ & $\begin{array}{l}0.116 \\
0.194\end{array}$ & $\begin{array}{l}0.64 \\
1.32\end{array}$ & $\begin{array}{l}0.075 \\
0.100\end{array}$ & $\begin{array}{l}0.646 \\
0.515\end{array}$ \\
\hline 196 & $\begin{array}{l}\text { Irpex lacteus } \\
*\end{array}$ & $\begin{array}{c}7 \\
14\end{array}$ & $\begin{array}{l}12.46 \\
15.65\end{array}$ & $\begin{array}{l}0.398 \\
0.404\end{array}$ & $\begin{array}{l}2.49 \\
2.01\end{array}$ & $\begin{array}{l}0.080 \\
0.052\end{array}$ & $\begin{array}{l}0.200 \\
0.128\end{array}$ \\
\hline 157 & $\begin{array}{l}\text { Lachnocladium } s p \text {. } \\
*\end{array}$ & $\begin{array}{c}7 \\
14\end{array}$ & $\begin{array}{l}12.86 \\
12.74\end{array}$ & $\begin{array}{l}0.331 \\
0.328\end{array}$ & $\begin{array}{l}1.83 \\
2.12\end{array}$ & $\begin{array}{l}0.047 \\
0.055\end{array}$ & $\begin{array}{l}0.142 \\
0.166\end{array}$ \\
\hline 072 & $\begin{array}{l}\text { Lentinula edodes } \\
*\end{array}$ & $\begin{array}{c}7 \\
14\end{array}$ & $\begin{array}{l}2.24 \\
4.70\end{array}$ & $\begin{array}{l}0.311 \\
0.331\end{array}$ & $\begin{array}{l}0.49 \\
1.18\end{array}$ & $\begin{array}{l}0.068 \\
0.083\end{array}$ & $\begin{array}{l}0.219 \\
0.250\end{array}$ \\
\hline 162 & Lentinus strigosus & $\begin{array}{c}7 \\
14\end{array}$ & $\begin{array}{l}4.74 \\
6.26\end{array}$ & $\begin{array}{l}0.373 \\
0.252\end{array}$ & $\begin{array}{l}0.11 \\
0.51\end{array}$ & $\begin{array}{l}0.009 \\
0.021\end{array}$ & $\begin{array}{l}0.023 \\
0.082\end{array}$ \\
\hline 268 & $\begin{array}{l}\text { Lentinus velutinus } \\
*\end{array}$ & $\begin{array}{c}7 \\
14\end{array}$ & $\begin{array}{l}6.37 \\
9.30\end{array}$ & $\begin{array}{l}0.344 \\
0.332\end{array}$ & $\begin{array}{l}1.46 \\
1.76\end{array}$ & $\begin{array}{l}0.079 \\
0.063\end{array}$ & $\begin{array}{l}0.229 \\
0.189\end{array}$ \\
\hline 110 & $\begin{array}{l}\text { Lepista } s p . \\
*\end{array}$ & $\begin{array}{c}7 \\
14\end{array}$ & $\begin{array}{r}5.82 \\
13.48\end{array}$ & $\begin{array}{l}0.485 \\
0.709\end{array}$ & $\begin{array}{l}2.68 \\
2.09\end{array}$ & $\begin{array}{l}0.223 \\
0.110\end{array}$ & $\begin{array}{l}0.460 \\
0.155\end{array}$ \\
\hline 279 & Macrolepiota procera & $\begin{array}{c}7 \\
14\end{array}$ & $\begin{array}{l}1.93 \\
3.94\end{array}$ & $\begin{array}{l}0.179 \\
0.281\end{array}$ & $\begin{array}{l}0.52 \\
0.88\end{array}$ & $\begin{array}{l}0.048 \\
0.063\end{array}$ & $\begin{array}{l}0.269 \\
0.223\end{array}$ \\
\hline 361 & $\begin{array}{l}\text { Marasmius cladophyllus } \\
*\end{array}$ & $\begin{array}{c}7 \\
14\end{array}$ & $\begin{array}{l}9.40 \\
9.91\end{array}$ & $\begin{array}{l}0.490 \\
0.312\end{array}$ & $\begin{array}{l}0.70 \\
0.56\end{array}$ & $\begin{array}{l}0.036 \\
0.018\end{array}$ & $\begin{array}{l}0.074 \\
0.056\end{array}$ \\
\hline
\end{tabular}


(continuação...)

\begin{tabular}{|c|c|c|c|c|c|c|c|}
\hline $\mathrm{CCB}$ & STRAIN & DAY & $\begin{array}{c}\mathrm{P}_{\mathrm{x}} \\
\text { g dry w./l }\end{array}$ & $\mathrm{Y}_{\mathrm{x} / \mathrm{s}}$ & $\begin{array}{c}\mathrm{P}_{\mathrm{p}} \\
\text { g dry w./1 } \\
\end{array}$ & $\mathrm{Y}_{\mathrm{p} / \mathrm{s}}$ & $\mathrm{Y}_{\mathrm{e}}$ \\
\hline 184 & $\begin{array}{l}\text { Melanoporia nigra } \\
*\end{array}$ & $\begin{array}{c}7 \\
14\end{array}$ & $\begin{array}{r}6.44 \\
12.16\end{array}$ & $\begin{array}{l}0.467 \\
0.316\end{array}$ & $\begin{array}{l}1.42 \\
1.90\end{array}$ & $\begin{array}{l}0.103 \\
0.049\end{array}$ & $\begin{array}{l}0.220 \\
0.156\end{array}$ \\
\hline 216 & $\begin{array}{l}\text { Nothopanus hygrophanus } \\
*\end{array}$ & $\begin{array}{c}7 \\
14\end{array}$ & $\begin{array}{l}16.16 \\
13.56\end{array}$ & $\begin{array}{l}0.444 \\
0.361\end{array}$ & $\begin{array}{l}1.78 \\
2.20\end{array}$ & $\begin{array}{l}0.049 \\
0.058\end{array}$ & $\begin{array}{l}0.110 \\
0.162\end{array}$ \\
\hline 164 & $\begin{array}{l}\text { Oligoporus } s p \text {. } \\
*\end{array}$ & $\begin{array}{c}7 \\
14\end{array}$ & $\begin{array}{r}4.54 \\
12.15\end{array}$ & $\begin{array}{l}0.286 \\
0.334\end{array}$ & $\begin{array}{l}2.71 \\
2.92\end{array}$ & $\begin{array}{l}0.170 \\
0.080\end{array}$ & $\begin{array}{l}0.597 \\
0.240\end{array}$ \\
\hline 179 & $\begin{array}{l}\text { Oudemansiella canarii } \\
*\end{array}$ & $\begin{array}{c}7 \\
14\end{array}$ & $\begin{array}{l}13.40 \\
15.37\end{array}$ & $\begin{array}{l}0.496 \\
0.430\end{array}$ & $\begin{array}{l}3.54 \\
1.70\end{array}$ & $\begin{array}{l}0.131 \\
0.048\end{array}$ & $\begin{array}{r}0.264 \\
0.111\end{array}$ \\
\hline 187 & Panaeolus papilionaceus & $\begin{array}{c}7 \\
14\end{array}$ & $\begin{array}{l}7.86 \\
5.91\end{array}$ & $\begin{array}{l}0.624 \\
0.296\end{array}$ & $\begin{array}{l}1.38 \\
1.70\end{array}$ & $\begin{array}{l}0.110 \\
0.085\end{array}$ & $\begin{array}{l}0.176 \\
0.288\end{array}$ \\
\hline 204 & $\begin{array}{l}\text { Peniophora cinerea } \\
*\end{array}$ & $\begin{array}{c}7 \\
14\end{array}$ & $\begin{array}{l}13.22 \\
13.72\end{array}$ & $\begin{array}{l}0.472 \\
0.352\end{array}$ & $\begin{array}{l}2.58 \\
3.04\end{array}$ & $\begin{array}{l}0.092 \\
0.078\end{array}$ & $\begin{array}{l}0.195 \\
0.222\end{array}$ \\
\hline 379 & $\begin{array}{l}\text { Perenniporia piperis } \\
*\end{array}$ & $\begin{array}{c}7 \\
14\end{array}$ & $\begin{array}{r}6.86 \\
12.06\end{array}$ & $\begin{array}{l}0.408 \\
0.520\end{array}$ & $\begin{array}{l}1.18 \\
1.90\end{array}$ & $\begin{array}{l}0.070 \\
0.082\end{array}$ & $\begin{array}{l}0.172 \\
0.158\end{array}$ \\
\hline 190 & $\begin{array}{l}\text { Phellinus gilvus } \\
*\end{array}$ & $\begin{array}{c}7 \\
14\end{array}$ & $\begin{array}{r}8.30 \\
10.32\end{array}$ & $\begin{array}{l}0.506 \\
0.266\end{array}$ & $\begin{array}{l}0.61 \\
1.23\end{array}$ & $\begin{array}{l}0.037 \\
0.032\end{array}$ & $\begin{array}{l}0.074 \\
0.119\end{array}$ \\
\hline 078 & $\begin{array}{l}\text { Pholiota nameko } \\
*\end{array}$ & $\begin{array}{c}7 \\
14\end{array}$ & $\begin{array}{l}2.96 \\
5.18\end{array}$ & $\begin{array}{l}0.302 \\
0.454\end{array}$ & $\begin{array}{l}1.64 \\
0.66\end{array}$ & $\begin{array}{l}0.167 \\
0.058\end{array}$ & $\begin{array}{l}0.554 \\
0.127\end{array}$ \\
\hline 394 & $\begin{array}{l}\text { Pleurotus flabellatus } \\
*\end{array}$ & $\begin{array}{c}7 \\
14\end{array}$ & $\begin{array}{l}5.94 \\
8.50\end{array}$ & $\begin{array}{l}0.381 \\
0.362\end{array}$ & $\begin{array}{l}0.81 \\
2.00\end{array}$ & $\begin{array}{l}0.052 \\
0.085\end{array}$ & $\begin{array}{l}0.136 \\
0.235\end{array}$ \\
\hline 004 & $\begin{array}{l}\text { Pleurotus ostreatus } \\
*\end{array}$ & $\begin{array}{c}7 \\
14\end{array}$ & $\begin{array}{l}4.06 \\
4.50\end{array}$ & $\begin{array}{r}0.366 \\
-\end{array}$ & $\begin{array}{l}0.57 \\
0.32\end{array}$ & $\begin{array}{r}0.051 \\
-\end{array}$ & $\begin{array}{l}0.140 \\
0.071\end{array}$ \\
\hline 016 & $\begin{array}{l}\text { Pleurotus ostreatoroseus } \\
*\end{array}$ & $\begin{array}{c}7 \\
14\end{array}$ & $\begin{array}{l}8.56 \\
9.00\end{array}$ & $\begin{array}{l}0.408 \\
0.280\end{array}$ & $\begin{array}{l}2.20 \\
2.38\end{array}$ & $\begin{array}{l}0.105 \\
0.074\end{array}$ & $\begin{array}{l}0.257 \\
0.264\end{array}$ \\
\hline 017 & $\begin{array}{l}\text { Pleurotus sajor-caju } \\
*\end{array}$ & $\begin{array}{c}7 \\
14\end{array}$ & $\begin{array}{l}11.47 \\
10.39\end{array}$ & $\begin{array}{l}0.484 \\
0.299\end{array}$ & $\begin{array}{l}1.85 \\
1.72\end{array}$ & $\begin{array}{l}0.078 \\
0.049\end{array}$ & $\begin{array}{l}0.161 \\
0.166\end{array}$ \\
\hline 001 & $\begin{array}{l}\text { Pleurotus sp. "florida" } \\
*\end{array}$ & $\begin{array}{c}7 \\
14\end{array}$ & $\begin{array}{l}11.02 \\
11.72\end{array}$ & $\begin{array}{l}0.510 \\
0.480\end{array}$ & $\begin{array}{l}2.85 \\
1.36\end{array}$ & $\begin{array}{l}0.132 \\
0.056\end{array}$ & $\begin{array}{l}0.259 \\
0.116\end{array}$ \\
\hline 259 & $\begin{array}{l}\text { Psilocybe castanella } \\
*\end{array}$ & $\begin{array}{c}7 \\
14\end{array}$ & $\begin{array}{l}8.96 \\
9.80\end{array}$ & $\begin{array}{l}0.498 \\
0.315\end{array}$ & $\begin{array}{l}1.18 \\
1.52\end{array}$ & $\begin{array}{l}0.066 \\
0.049\end{array}$ & $\begin{array}{l}0.132 \\
0.155\end{array}$ \\
\hline 224 & Psilocybe subcubensis & $\begin{array}{c}7 \\
14\end{array}$ & $\begin{array}{l}2.92 \\
4.96\end{array}$ & $\begin{array}{l}0.243 \\
0.359\end{array}$ & $\begin{array}{l}0.58 \\
0.59\end{array}$ & $\begin{array}{l}0.048 \\
0.043\end{array}$ & $\begin{array}{l}0.199 \\
0.119\end{array}$ \\
\hline 113 & Pycnoporus sanguineus & $\begin{array}{c}7 \\
14\end{array}$ & $\begin{array}{l}6.10 \\
7.83\end{array}$ & $\begin{array}{l}0.295 \\
0.201\end{array}$ & $\begin{array}{l}1.04 \\
0.87\end{array}$ & $\begin{array}{l}0.050 \\
0.022\end{array}$ & $\begin{array}{l}0.170 \\
0.111\end{array}$ \\
\hline 277 & Pycnoporus sanguineus & $\begin{array}{c}7 \\
14\end{array}$ & $\begin{array}{l}0.36 \\
0.43\end{array}$ & $\begin{array}{l}0.047 \\
0.090\end{array}$ & $\begin{array}{l}0.74 \\
0.82\end{array}$ & $\begin{array}{l}0.096 \\
0.171\end{array}$ & $\begin{array}{l}2.056 \\
1.907\end{array}$ \\
\hline 334 & $\begin{array}{l}\text { Rigidoporus microporus } \\
*\end{array}$ & $\begin{array}{c}7 \\
14\end{array}$ & $\begin{array}{r}4.70 \\
16.04\end{array}$ & $\begin{array}{l}0.402 \\
0.685\end{array}$ & $\begin{array}{l}0.81 \\
0.83\end{array}$ & $\begin{array}{l}0.069 \\
0.035\end{array}$ & $\begin{array}{l}0.172 \\
0.052\end{array}$ \\
\hline
\end{tabular}


(continuação...)

\begin{tabular}{|c|c|c|c|c|c|c|c|}
\hline$\overline{\mathrm{CCB}}$ & STRAIN & $\overline{\mathrm{DAY}}$ & $\begin{array}{c}\mathrm{P}_{\mathrm{x}} \\
\text { g dry w./l }\end{array}$ & $Y_{x / s}$ & $\begin{array}{c}\mathrm{P}_{\mathrm{p}} \\
\text { g dry w./l }\end{array}$ & $\mathrm{Y}_{\mathrm{p} / \mathrm{s}}$ & $\mathrm{Y}_{\mathrm{e}}$ \\
\hline 467 & $\begin{array}{l}\text { Ripartitella } \mathrm{cf} \text {. brasiliensis } \\
*\end{array}$ & $\begin{array}{c}7 \\
14\end{array}$ & $\begin{array}{r}6.15 \\
11.80\end{array}$ & $\begin{array}{l}0.521 \\
0.371\end{array}$ & $\begin{array}{l}0.86 \\
1.50\end{array}$ & $\begin{array}{l}0.073 \\
0.047\end{array}$ & $\begin{array}{l}0.140 \\
0.127\end{array}$ \\
\hline 368 & Schizophyllum commune & $\begin{array}{c}7 \\
14\end{array}$ & $\begin{array}{l}7.22 \\
6.02\end{array}$ & $\begin{array}{l}0.185 \\
0.154\end{array}$ & $\begin{array}{l}1.85 \\
0.91\end{array}$ & $\begin{array}{l}0.047 \\
0.023\end{array}$ & $\begin{array}{l}0.256 \\
0.151\end{array}$ \\
\hline 473 & Schizophyllum commune & $\begin{array}{c}7 \\
14\end{array}$ & $\begin{array}{l}13.84 \\
16.68\end{array}$ & $\begin{array}{l}0.416 \\
0.429\end{array}$ & $\begin{array}{l}1.32 \\
1.76\end{array}$ & $\begin{array}{l}0.040 \\
0.045\end{array}$ & $\begin{array}{l}0.095 \\
0.106\end{array}$ \\
\hline 474 & Schizophyllum commune & $\begin{array}{c}7 \\
14\end{array}$ & $\begin{array}{l}10.84 \\
16.10\end{array}$ & $\begin{array}{l}0.386 \\
0.415\end{array}$ & $\begin{array}{l}1.01 \\
1.97\end{array}$ & $\begin{array}{l}0.036 \\
0.051\end{array}$ & $\begin{array}{l}0.093 \\
0.122\end{array}$ \\
\hline 202 & $\begin{array}{l}\text { Trametes versicolor } \\
*\end{array}$ & $\begin{array}{c}7 \\
14\end{array}$ & $\begin{array}{r}10.20 \\
7.43\end{array}$ & $\begin{array}{l}0.313 \\
0.191\end{array}$ & $\begin{array}{l}1.51 \\
2.34\end{array}$ & $\begin{array}{l}0.046 \\
0.060\end{array}$ & $\begin{array}{l}0.148 \\
0.315\end{array}$ \\
\hline 165 & $\begin{array}{l}\text { Trametes villosa } \\
*\end{array}$ & $\begin{array}{c}7 \\
14\end{array}$ & $\begin{array}{r}6.86 \\
10.07\end{array}$ & $\begin{array}{l}0.279 \\
0.259\end{array}$ & $\begin{array}{l}1.73 \\
2.65\end{array}$ & $\begin{array}{l}0.070 \\
0.068\end{array}$ & $\begin{array}{l}0.252 \\
0.263\end{array}$ \\
\hline 213 & $\begin{array}{l}\text { Trametes villosa } \\
*\end{array}$ & $\begin{array}{c}7 \\
14\end{array}$ & $\begin{array}{l}7.24 \\
9.02\end{array}$ & $\begin{array}{l}0.234 \\
0.232\end{array}$ & $\begin{array}{l}1.99 \\
2.12\end{array}$ & $\begin{array}{l}0.064 \\
0.055\end{array}$ & $\begin{array}{l}0.275 \\
0.235\end{array}$ \\
\hline 203 & $\begin{array}{l}\text { Trichaptum byssogenum } \\
*\end{array}$ & $\begin{array}{c}7 \\
14\end{array}$ & $\begin{array}{l}9.00 \\
7.86\end{array}$ & $\begin{array}{l}0.232 \\
0.203\end{array}$ & $\begin{array}{l}1.35 \\
1.48\end{array}$ & $\begin{array}{l}0.035 \\
0.038\end{array}$ & $\begin{array}{l}0.150 \\
0.188\end{array}$ \\
\hline 082 & $\begin{array}{l}\text { Tricholoma crassum } \\
*\end{array}$ & $\begin{array}{c}7 \\
14\end{array}$ & $\begin{array}{l}11.15 \\
15.90\end{array}$ & $\begin{array}{l}0.791 \\
0.646\end{array}$ & $\begin{array}{l}2.20 \\
3.23\end{array}$ & $\begin{array}{l}0.156 \\
0.131\end{array}$ & $\begin{array}{l}0.197 \\
0.203\end{array}$ \\
\hline 390 & $\begin{array}{l}\text { Trogia buccinalis } \\
*\end{array}$ & $\begin{array}{c}7 \\
14\end{array}$ & $\begin{array}{l}5.74 \\
7.86\end{array}$ & $\begin{array}{l}0.279 \\
0.273\end{array}$ & $\begin{array}{l}1.03 \\
1.60\end{array}$ & $\begin{array}{l}0.050 \\
0.056\end{array}$ & $\begin{array}{l}0.179 \\
0.204\end{array}$ \\
\hline 193 & Tyromyces pseudolacteus & $\begin{array}{c}7 \\
14 \\
\end{array}$ & $\begin{array}{l}8.34 \\
6.50 \\
\end{array}$ & $\begin{array}{l}0.323 \\
0.227 \\
\end{array}$ & $\begin{array}{l}1.19 \\
0.77\end{array}$ & $\begin{array}{l}0.046 \\
0.027\end{array}$ & $\begin{array}{l}0.143 \\
0.118\end{array}$ \\
\hline
\end{tabular}

* Formation of gel due to the freezing of the culture filtrate

$\mathrm{P}_{\mathrm{x}}=\mathrm{g}$ dry weight biomass $/ 1$ culture

$\mathrm{Y}_{\mathrm{x} / \mathrm{s}}=\mathrm{g}$ dry weight biomass/ $\mathrm{g}$ consumed glucose

\section{RESULTS AND DISCUSSION}

Almost all the strains produced exopolysaccharide in different quantities (Table 1). The best yield was produced by Agaricus sp., with $6.01 \mathrm{~g}$ dry w./1 (conversion yield, $\mathrm{Y}_{\mathrm{p} / \mathrm{s}}=0.761$ ) and Oudemansiella canarii with $3.54 \mathrm{~g}$ dry w./1 $\left(\mathrm{Y}_{\mathrm{p} / \mathrm{s}}=0.131\right)$ after 7 days of incubation. Tricholoma crassum had a similar production (3.23 g dry w./l) with conversion yield of 0.131 , but after 14 days of incubation.

About $30 \%$ of the strains produced more exopolysaccharide after 7 days incubation; however $70 \%$ produced more after 14 days, which indicates that an accurate study of each strain with growth and
$\mathrm{P}_{\mathrm{p}}=\mathrm{g}$ dry weight biopolymer/ 1 culture

$\mathrm{Y}_{\mathrm{p} / \mathrm{s}}=\mathrm{g}$ dry weight biopolymer/g consumed glucose $\mathrm{Y}_{\mathrm{e}}=$ specific yield

product kinetic profiles should be carried out if there is a possibility of its utilization for polymer production. The strains that produced more than $2.0 \mathrm{~g}$ dry w./1 of exopolysaccharide are shown in Fig. 1.

There is no relation between biomass and exopolysaccharide production and in some cases a considerable decrease of biopolymer was observed after 14 days incubation (Agaricus sp., Calvatia cyathiformis, Oudemansiella canarii and Pleurotus sp. "florida").

The conversion yield of glucose as polymer varied between 0.020 and 0.100 for $75 \%$ of the strains and the best yields were those of Agaricus sp. (0.761) and Calvatia cyathiformis (0.293). 
Figure 1. Strains that produced more than $2.0 \mathrm{~g}$ dry w./l of polymer after 7 and 14 days.
國 exopolys accharide
$\square$ biomass

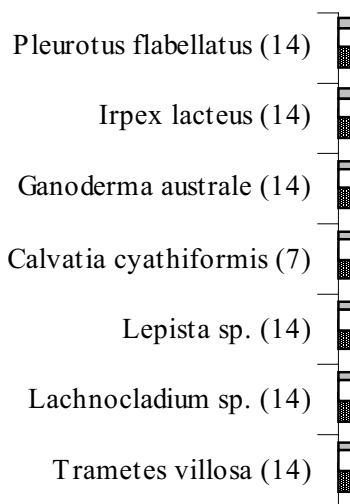

Nothopanus hygrophanus (14)

Pleurotus ostreatoroseus (7)

Tricholoma crassum (7)

Trametes versicolor (14)

Pleurotus ostreatoroseus (14)

$$
\text { Irpex lacteus (7) }
$$

Peniophora cinerea (7)

Ganoderma australe (7)

Trametes villosa (14)

Lepista sp. (7)

Oligoporus sp. (7)

Ganoderna lipsiensis (14)

Pleurotus sp. "florida" (7)

Oligoporus sp. (14)

Peniophora cinerea (14)

Tricholoma crassum (14)

Oudemansiella canarii (7)

Agaricus sp. (7)
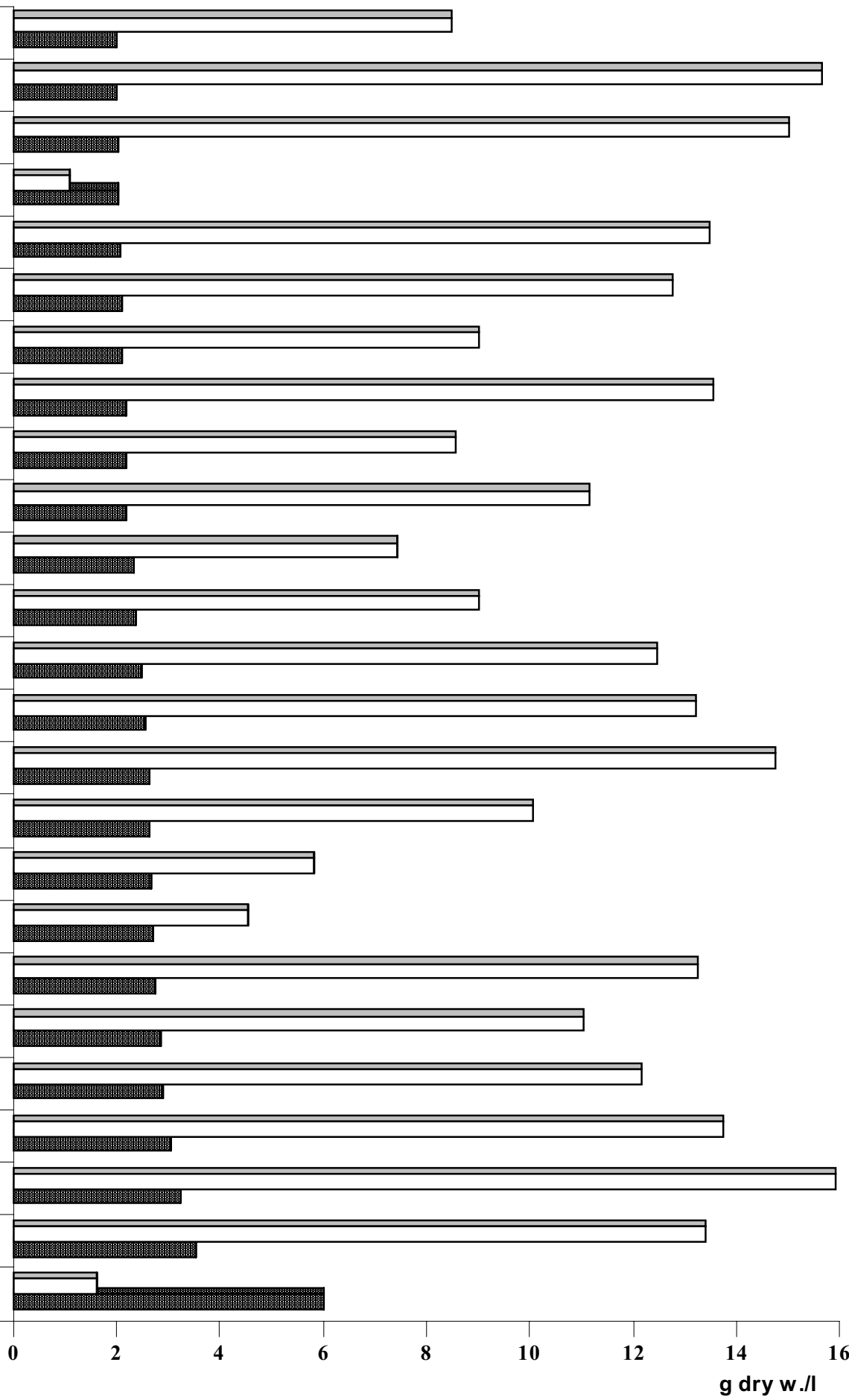
Different strains of Schizophyllum commune, Pycnoporus sanguineus and Trametes villosa showed different results not only for biomass, but also for polymer production. These data confirm the diversity of exopolysaccharide production among different strains in submerged culture. Another strain of Schizophyllum commune was submitted to a similar screening by Cavazzoni and Adami (4) with the same growing conditions used here. The polymer production was higher (5.3 g dry w./1).

An interesting observation was made concerning the formation of an insoluble gel when the culture filtrate was frozen prior to polysaccharide precipitation. In the Table 1 these strains are marked. This peculiar characteristic could aid polymer separation, since there is no need of an organic solvent such as isopropanol, ethanol or acetone for the precipitation of the polymer, thus increasing the process viability. Moreover, it is important to observe that the product obtained by solvent precipitation cannot be considered pure polysaccharide because proteins and salts present in the medium coprecipitate. The data obtained from this screening are just indicative for selecting strains for further investigations on exopolysaccharide production.

Some of the strains studied here were submitted to a lignin degradation activity test (2). All strains that produced more than $2.0 \mathrm{~g}$ dry w./l of exopolysaccharide showed good lignin degradation activity (24.8-65.4\%) at $25^{\circ} \mathrm{C}$ and 60 days of incubation. For Irpex lacteus the result was higher at $30^{\circ} \mathrm{C}$ with $78.4 \%$ of substrate lignin degradation. Okino (15) studied some of these strains for laccase and peroxidase production and all of them showed enzyme activity.

Biomass production ranged from 0.34 to $16.68 \mathrm{~g}$ dry w./l. Some strains, such as Agaricus xanthodermus, Calvatia cyathiformis and Climacodon pulcherrimus, had a slow growth rate in these culture conditions. Others, such as Schyzophyllum commune, Rigidoporus microporus, Oudemansiella canarii, Irpex lacteus and Nothopanus hygrophanus produced more than 15.00 $\mathrm{g}$ dry w./l of biomass.

Among the edible strains, those that produced more biomass after 7 days incubation were Pleurotus sajor-caju (11.47 g dry w./l), Pleurotus sp. "florida" (11.02 g dry w./l), and Agrocybe platensis (10.18 g dry w./l). After 14 days incubation, the best biomass producer was Lepista sp. (13.48 g dry w./l).

The conditions used for the submerged culture could be considered adequate for biomass production.
Data presented in literature $(1,5,11,12)$ showed lower production for Pleurotus species with other culture parameters.

During estimation of polymer and biomass produced it is important to consider that exopolysaccharides adherent to the hyphae are also entrapped into the pellets formed during the submerged culture (1), which means that the dry weight of biopolymer which precipitated from the culture filtrate does not correspond to the total exopolysaccharide and that the biomass can be overestimated. To minimize this problem biomass was washed twice with distilled water.

During the screening it was observed that the submerged cultures showed different characteristics according to the fungal species. The pellets formed can be regular or irregular in form and size. The form varies from spherical to cylindrical and the size from 1 to $20 \mathrm{~mm}$. In some cases the formation of pellets was not observed, but rather a mycelial agglomeration without a defined form (13).

The pellets were smooth, hairy (with looser outer zones) or with fringes of aggregated hyphae that give the pellet a star form. The color and consistency were also different, as well as the flavour. In the case of Auricularia fuscosuccinea the pellet had a gelatinous consistency. Sometimes the culture filtrate was very clear, other times was turbid and very viscous. In most of the cultures the presence of crystals with different forms was observed, which could indicate, in some cases, the presence of excreted metabolites.

When there is a depletion of glucose in the medium it was observed that pellets begin to become darker and break up. The dead hyphae are decomposed and the resulting substances are reabsorbed by the mycelium.

Results showed that most of the Basidiomycetes strains screened are potential exopolysaccharide producers. The possibility of using these biopolymers for medical application promises a large opportunity to improve the study of such group of fungi. Besides the Brazilian mycobiota has been scarcely investigated although its great potentiality.

\section{ACKNOWLEDGMENTS}

This work was supported by National Council of Research of Italy, Coordinating Project of Polysaccharides. The authors are also grateful to $\mathrm{CNPq} /$ Brasilia - Brazil (Brazilian Education Ministry) for financial support. 


\section{RESUMO}

\section{Triagem de basidiomicetos para a produção de exopolissacarídeos e biomassa em cultura líquida}

Este trabalho diz respeito à produção de exopolissacarídeos e biomassa por basidiomicetos em cultura líquida. O "screening" foi realizado com 56 linhagens incluindo fungos nativos de diferentes ecossistemas do Brasil e de fungos comestíveis. Agaricus sp. (CCB 280) e Oudemansiella canarii (Jungh.) Hohn (CCB 179) foram os melhores produtores de exopolissacarídeo (6,01 e 3,54 g peso seco/l respectivamente), em 7 dias de incubação. $\mathrm{O}$ melhor produtor de biomassa foi Schizophyllum commune Fr::Fr. (CCB 473) com 16,68 g peso seco/l em 14 dias de incubação. Quando o filtrado cultural foi submetido à congelamento antes da precipitação do polissacarídeo, formou-se uma fração gelatinosa.

Palavras-chave: Basidiomiceto, exopolissacarídeo, biomassa, cultura líquida

\section{REFERENCES}

1. Burns, P.J.; Yeo, P.; Keshavarz, T.; Roller, S.; Evans, C.S Physiological studies of exopolysaccharide production from the Basidiomycete Pleurotus florida. Enzyme Microb. Technol. 16:566-572, 1994

2. Capelari, M.; Zadrazil, F. Lignin degradation and in vitro digestibility of wheat straw treated with Brazilian Tropical species of white rot fungi. Folia Microbiol., 42:481-487, 1997.

3. Catley, B.J. The biochemistry of some fungal polysaccharides with industrial potencial. In: Arora, D.K.; Elander, R.P.; Mukerji, K.G. (eds.) Handbook of applied mycology: fungal biotechnology. Marcel Dekker, New York, 1992, p.1114.
4. Cavazzoni, V.; Adami, A. Exopolysaccharides produced by mycelial edible mushrooms. Ital. J. of Food Sci., 1:9-15, 1992.

5. Compere, A.L.; Griffith, W.L.; Greene, S.V. Polymer production by Pleurotus. Dev. Ind. Microbiol., 21:461-469, 1980.

6. Gutiérrez, A. Exopolisacaridos y metabolitos aromaticos de Pleurotus: naturaleza y función en la degradación de la lignina. Sevilla, 1995, 121 p. (Ph.D. Thesis Facultad de Farmacia, Universidad de Sevilla).

7. Hamlyn, P.F.; Schmidt, R.J. Potential therapeutic application of fungal filaments in wound management. Mycologist, 8:147-152, 1994.

8. Jong, S.C.; Birgmingham, J.M. Medicinal benefits of the mushroom Ganoderma. Adv. Appl. Microbiol., 37:101-134, 1992.

9. Jong, S.C.; Birgmingham, J.M. Medicinal and therapeutic value of the shiitake mushroom. Adv. Appl. Microbiol., 39:153-184, 1993.

10. Jong, S.C.; Birgmingham, J.M. Mushrooms as a source of natural flavour and aroma compounds. In: Chang., S.T; Buswell, J.A; Chiu, S.W. (eds.) Mushroom biology and mushroom products. The Chinese University Press, Hong Kong, 1993, p.345-366.

11. Manachini, P.L. Screening di funghi superiori commestibili per la produzione di biomasse e di metabolici esocellulari in coltura sommersa. Tecnol. Alim. - Setembre/Otobre:17-24, 1979.

12. Masaphy, S.; Levanon, D. The effect of lignocellulose on lignocellulolytic activity of Pleurotus pulmonarius in submerged culture. Appl. Microbiol. Biotechnol., 36:828-832, 1992.

13. Maziero, R. Produção de exopolissacarídeos por basidiomicetos em cultura submersa: "screening", caracterização química preliminar e estudo de produção utilizando Irpex lacteus (Fr.:Fr.) Fr. São Paulo, 1996, 181p. (Ph.D. Thesis. Instituto de Biociências, USP).

14. Maziero, R.; Adami, A.; Cavazzoni, V.; Bononi, V.L. Exopolysaccharide and biomass production in submerged culture by edible mushrooms. Mush. Science XIV:887-892, 1995.

15. Okino, L.K. Atividade lignolítica de basidiomicetos brasileiros. Rio Claro, 1996, 58p. (Master Dissertation. Instituto de Biociências de Rio Claro, UNESP).

16. Ruel, K.; Joseleau, J.-P. Involvement of an extracellular glucan sheat during degradation of Populus wood by Phanerochaete chrysosporium. Appl. Environ. Microbiol. 57:374-384, 1991.

17. Somogyi, M. Notes on sugar determination. J. Biol. Chem., 195:19-23, 1952 